\title{
Exploring the Evidence of Expanded Criteria for Endoscopic Resection of Early Gastric Cancers
}

\author{
II Ju Choi \\ Department of Gastroenterology, Center for Gastric Cancer, National Cancer Center, Goyang, Korea
}

See "Comparison of Endoscopic Submucosal Dissection and Surgery for Differentiated Type Early Gastric Cancer within the Expanded Criteria” by Dong Woo Shin, Hee Young Hwang and Seong Woo Jeon, on page 170-178.

Traditionally, gastrectomy with lymph node dissection is recommended as a curative treatment modality. In Korea, gastric cancer screening is covered by the Health Insurance System for individuals aged 40 years or older; therefore, gastric cancer is now detected in its early stages. Minimally invasive treatment using endoscopic resection is indicated for early gastric cancer with a low risk of lymph node metastasis. ${ }^{1}$ As proposed by the Japanese Gastric Cancer Association, endoscopic resection is now the accepted standard treatment if the gastric cancer fulfills the following absolute criteria: $\leq 2 \mathrm{~cm}$ tumor size, intramucosal, differentiated histological type of cancer, and absence of ulceration. ${ }^{1}$ Current guidelines suggest that the criteria can be broadened to include larger lesions $(>3$ $\mathrm{cm})$, ulcerative lesions of relatively small size $(\leq 3 \mathrm{~cm})$, superficial submucosal invasive cancer $(\leq 500 \mu \mathrm{m}) \leq 3 \mathrm{~cm}$ in size, or undifferentiated cancer within $2 \mathrm{~cm}$ in size if other conditions are compatible with the absolute criteria. ${ }^{1,2}$

The current guidelines define the expanded indication for endoscopic submucosal dissection (ESD) as an investigational treatment because the existing evidence is still insufficient for its use as a curative treatment modality. ${ }^{1}$ Two possible ap-

Received: March 11, 2017 Revised: March 21, 2017

Accepted: March 21, 2017

Correspondence: Il Ju Choi

Department of Gastroenterology, Center for Gastric Cancer, National Cancer Center, 323 Ilsan-ro, Ilsandong-gu, Goyang 10408, Korea

Tel: +82-31-920-2282, Fax: +82-31-920-0069, E-mail: cij1224@ncc.re.kr

(cc) This is an Open Access article distributed under the terms of the Creative Commons Attribution Non-Commercial License (http://creativecommons.org/ licenses/by-nc/3.0) which permits unrestricted non-commercial use, distribution, and reproduction in any medium, provided the original work is properly cited. proaches can be used to prove the acceptability of an expanded indication. The first is to show that the long-term survival rates in the expanded indication are comparable with those of the absolute indication, in which ESD is a standard treatment. Several large-series retrospective studies have shown that long-term outcomes of the two indications were quite similar and excellent. ${ }^{3,4}$ The second approach is to prove that the outcomes of the expanded indication are comparable or better than those of the standard surgical treatment (i.e., gastrectomy with lymph node dissection). ${ }^{5}$ Prospective randomized trial will be the best study design but is virtually impossible to perform. Therefore, an approach that uses retrospective comparative analyses might provide an acceptable option.

In this issue of Clinical Endoscopy, Shin et al. reported a confirmative study showing that long-term survival after ESD was not significantly different from that after gastrectomy. ${ }^{6}$ The study analyzed 275 propensity score-matched patients with a median follow-up duration of 56 months and showed that the 5-year overall survival rate was $92.0 \%$ in the ESD group and $93.3 \%$ in the gastrectomy group. Moreover, the complication rates were much lower after ESD (5.1\%) than after gastrectomy (15.0\%). The reported results were similar to those of recent studies that showed a comparable long-term outcome of ESD in terms of overall survival rate with that of surgery, including lower complication rates. ${ }^{5,78}$ The length of hospital stay and the treatment costs in ESD treatment were superior to those in surgery for early gastric cancer within the expanded indication, as previously reported. ${ }^{9}$

When interpreting the results of this study, several limitations should be emphasized. First, the study suggested a 
possible selection bias due to the retrospective study design. Patients who were lost to follow-up within 36 months after treatment were excluded from the analysis but comprised approximately $30 \%$ of the ESD group and $25 \%$ of the gastrectomy group. These patients can have different characteristics from those included in the analysis, such as poorer general health, older age, or severe comorbidity that might affect overall survival.

Second, the control of possible confounding factors was a challenge when analyzing the retrospective data. Several techniques are available to correct this problem, including the use of multivariable logistic regression, propensity score adjustment, propensity score matching, or propensity score-based weighting analyses. ${ }^{10}$ However, the results of using these various methods could lead to opposite conclusions, even within the same data set, as was shown in a German stroke registry analysis that evaluated the effect of tissue plasminogen activator on stroke mortality. ${ }^{10}$ In the study of Shin et al., the propensity score-matching method was not clearly described because the ratio of the included patients who underwent ESD was 1.75 in comparison with that in the gastrectomy group. ${ }^{6}$ Moreover, many baseline characteristics differed between the groups, including lesion size, morphological type, and presence of ulceration.

Another important limitation of this study was its small sample size, especially in the expanded criteria (51 individuals in the ESD group and 29 in the gastrectomy group), which comprised approximately $30 \%$ of all included patients. Therefore, the results of this study were highly dependent on the absolute indication rather than on the expanded indication, and could not be generalized to the expanded indication.

Although the main findings of this study have been confirmed, as previously reported, ${ }^{5,8}$ some important points should be emphasized in this study. First, two mortality cases were reported in the gastrectomy group. Procedure-related mortality is rare with ESD, but the surgical mortality rates after laparoscopic or open distal gastrectomy for stage I gastric cancers were $0.6 \%$ and $0.3 \%$, respectively. ${ }^{11}$ The criteria for endoscopic resection were usually accepted for lesions with very low lymph node metastasis risk; therefore, the upper limit of lymph node metastasis risk in surgical specimens was $<1 \%$. ${ }^{12}$ These suggestions might be reasonable, but whether this is a fair criterion for selecting candidates for endoscopic resection should be determined. It is noteworthy that the mortality after surgical treatment in this study was $2 \%(2 / 100)$, and its upper limit far exceeded $1 \%$. Even with the presence of lymph node metastasis, the patient should not be considered a mortality case because a surgical option is available for curative treatment.

Expanded indications could include undifferentiated can- cer. However, this study did not include this specific criterion but instead focused on the differentiated histological type. ${ }^{6}$ The undifferentiated histological type of cancer was reported to have a lower curative resection rate in short-term outcome analyses of ESD. ${ }^{13,14}$ The long-term outcome for this group of patients should be evaluated separately in future studies.

Preoperative evaluations of early gastric cancer in terms of size, depth, presence of ulceration, and lymphovascular invasion status are difficult and have inevitable discrepancies. Therefore, approximately 15\%-20\% of gastric cancer cases treated with ESD was inappropriate. However, many of the patients with out-of-indication cases refused to undergo an additional operation and had lower survival rates than those who were initially treated with surgery. ${ }^{15}$ Retrospective studies usually excluded these patients because of loss to follow-up. This might result in a selection bias by excluding patients who were expected to have a poor prognosis. Thus, the comparable long-term outcomes after ESD based on the post-treatment pathology cannot provide direct evidence of comparable outcomes for preoperative expanded indications.

In the future, overall and disease-specific survival rates are parameters that should be considered in the study on the expanded ESD criteria. Although prospective randomized trials could provide more information, it may be difficult to perform such studies. Well-designed retrospective and prospective cohort studies are needed to determine if ESD treatment can be accepted as a standard treatment for expanded indications by ending its status as an investigational treatment.

\section{Conflicts of Interest}

The author has no financial conflicts of interest.

\section{REFERENCES}

1. Japanese Gastric Cancer Association. Japanese gastric cancer treatment guidelines 2014 (ver. 4). Gastric Cancer 2017;20:1-19.

2. Ono H, Yao K, Fujishiro M, et al. Guidelines for endoscopic submucosal dissection and endoscopic mucosal resection for early gastric cancer. Dig Endosc 2016;28:3-15.

3. Suzuki H, Oda I, Abe S, et al. High rate of 5-year survival among patients with early gastric cancer undergoing curative endoscopic submucosal dissection. Gastric Cancer 2016;19:198-205.

4. Ahn JY, Jung HY, Choi KD, et al. Endoscopic and oncologic outcomes after endoscopic resection for early gastric cancer: 1370 cases of absolute and extended indications. Gastrointest Endosc 2011;74:485-493.

5. Kim YI, Kim YW, Choi IJ, et al. Long-term survival after endoscopic resection versus surgery in early gastric cancers. Endoscopy 2015;47:293301.

6. Shin DW, Hwang HY, Jeon SW. Comparison of endoscopic submucosal dissection and surgery for differentiated type early gastric cancer within the expanded criteria. Clin Endosc 2017;50:170-178.

7. Choi IJ, Lee JH, Kim YI, et al. Long-term outcome comparison of endoscopic resection and surgery in early gastric cancer meeting the absolute indication for endoscopic resection. Gastrointest Endosc 2015;81:333341.e1. 
8. Pyo JH, Lee $\mathrm{H}, \mathrm{Min} \mathrm{BH}$, et al. Long-term outcome of endoscopic resection vs. surgery for early gastric cancer: a non-inferiority-matched cohort study. Am J Gastroenterol 2016;111:240-249.

9. Kim Y, Kim YW, Choi IJ, et al. Cost comparison between surgical treatments and endoscopic submucosal dissection in patients with early gastric cancer in Korea. Gut Liver 2015;9:174-180.

10. Kurth T, Walker AM, Glynn RJ, et al. Results of multivariable logistic regression, propensity matching, propensity adjustment, and propensity-based weighting under conditions of nonuniform effect. Am J Epidemiol 2006;163:262-270.

11. Kim W, Kim HH, Han SU, et al. Decreased morbidity of laparoscopic distal gastrectomy compared with open distal gastrectomy for stage I gastric cancer: short-term outcomes from a multicenter randomized controlled trial (KLASS-01). Ann Surg 2016;263:28-35.

12. Gotoda T, Yanagisawa A, Sasako M, et al. Incidence of lymph node metastasis from early gastric cancer: estimation with a large number of cases at two large centers. Gastric Cancer 2000;3:219-225.

13. Abe S, Oda I, Suzuki H, et al. Short- and long-term outcomes of endoscopic submucosal dissection for undifferentiated early gastric cancer. Endoscopy 2013;45:703-707.

14. Kim JH, Lee YC, Kim H, et al. Endoscopic resection for undifferentiated early gastric cancer. Gastrointest Endosc 2009;69:e1-e9.

15. Eom BW, Kim YI, Kim KH, et al. Survival benefit of additional surgery after noncurative endoscopic resection in patients with early gastric cancer. Gastrointest Endosc 2017;85:155-163.e3. 